\title{
A Systematic Review of the Use of Telepsychiatry in Depression
}

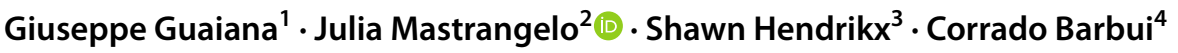

Received: 28 April 2020 / Accepted: 3 October 2020 / Published online: 10 October 2020

(c) Springer Science+Business Media, LLC, part of Springer Nature 2020

\begin{abstract}
Telepsychiatry, the use of televideo in psychiatric assessment and treatment, is utilized throughout Canada. Major depressive disorder (MDD) is common, with significant burdens of suffering and cost. This systematic review explores the literature on the use of televideo to diagnose and treat MDD, particularly acceptability and patient satisfaction, efficacy, and costeffectiveness. A literature search was conducted for years 1946 to 2019. Study eligibility criteria included: MDD as the condition of interest, use of televideo technology, randomized controlled trials (RCTs), Adult (18 years or older) population, any clinical setting, and any healthcare professional providing care. The study must have included at least one of the following measures, satisfaction, efficacy, and cost-effectiveness. Fourteen studies were included. Satisfaction is equivalent to or significantly higher than face-to-face intervention. Both televideo and control groups found relief from depressive symptoms, with differences either statistically insignificant or in favour of televideo. Despite increased cost upfront for televideo due to the technology required, televideo would eventually be more cost-effective due to reducing travel expenses. Limitations include that there is little RCT data, and what exists often uses a collaborative treatment model. Many studies consisted solely of U.S. Veterans, and have limited generalizability. Further research needed to directly compare psychiatrist assessment over televideo versus in-person, and determine if particular patient subgroups benefit more from televideo or in-person intervention.
\end{abstract}

Systematic review registration number: CRD42016048224.

Keywords Telemedicine $\cdot$ Telepsychiatry $\cdot$ Major depressive disorder

\section{Introduction}

Telemedicine, the ability to provide healthcare remotely via technology, has the potential to reinvent the practice of medicine. This technology typically consists of a live video and audio connection between a physician and a patient (who may be located in a hospital, clinic, or their own home), provided by a service, which can be private or

Giuseppe Guaiana

gguaiana@stegh.on.ca

1 Department of Psychiatry and Department of Epidemiology and Biostatistics, Western University, London, Canada

2 Department of Psychiatry, Western University, London, Canada

3 Collections \& Content Strategies, Western Libraries, Western University, London, Canada

4 Department of Neurosciences, Biomedicine and Movement Sciences, Section of Psychiatry, University of Verona, Verona, Italy government-based. Patients in rural areas, those unable to leave their homes, or who must navigate other barriers, can access care via telemedicine they otherwise could not (Chaet et al. 2017). In their annual report for the years 2017-2018, the Ontario Telemedicine Network ([OTN], the governmentbased telemedicine network for the Canadian province of Ontario), reported facilitating 896529 patient consultations, which they estimate saved $\$ 71.9$ million in Northern Health Travel Grants, and allowed patients to avoid travelling 270 million km (OTN 2018).

Psychiatry appears particularly well-suited to this model, as an assessment consists mainly of speaking with and laying eyes on the patient, both of which can be accomplished by telepsychiatry (the use of telemedicine to provide psychiatric assessment and treatment; Lambert and Wertheimer 2016). Telepsychiatry appears to be helpful in terms of providing care to rural patients, as $45 \%$ of the telepsychiatry consultations were for patients in a northern or rural area (Serhal et al. 2017). Australia, similarly, has a relatively small population spread out over a large geographical area, 
and their healthcare system is partially publicly-funded, making them a reasonable comparison for Canada. Interestingly, 55.8\% of their psychiatrists surveyed practiced using telepsychiatry, and this increased to $81.4 \%$ among psychiatrists who indicated their patient base was in remote areas (Royal Australian and New Zealand College of Psychiatrists [RANZCP] 2013).

Major depressive disorder (MDD) is a common disorder with significant associated burden in terms of patient suffering and societal cost. The annual prevalence of a major depressive episode (MDE) in Canada is approximately $4.7 \%$, with a lifetime prevalence of $11.3 \%$ (Canadian Network for Mood and Anxiety Treatments [CANMAT] 2016).

Clearly, it is important for the health of patients and our communities to remove barriers that may prevent patients from accessing the healthcare they need. The emergence of the coronavirus disease in 2019 (Cevik et al. 2020) has made the assessment of remote technology even more pressing.

Evidence supporting the use of telemedicine in depression is still uncertain (García-Lizana and Muñoz-Mayorga 2010) and it will be important to assess whether this modality of delivering case is safe, effective and accepted by patients.

The goal of this review is to provide a systematic review on telepsychiatry in MDD and assess its face validity, acceptability by patients and cost-effectiveness compared to in-person care, so that providers will be better equipped to understand its advantages and potential drawbacks.

\section{Methodology}

\section{Protocol and Registration}

The protocol of the study was published on PROSPERO (https://www.crd.york.ac.uk/PROSPERO/displ ay_record.php?ID=CRD42016048224; record ID: CRD42016048224).

\section{Eligibility Criteria}

The inclusion criteria were:

1. Unipolar depression (major depressive disorder) as the condition of interest

2. Use of some form of tele-technology

3. Randomized controlled trials (RCTs)

4. Adult (18 years or older) population

5. Any clinical setting (i.e. in hospital, outpatient)

6. Any healthcare professional providing care (i.e. psychiatrists, family physicians, nurses, psychologists, etc.)

7. Written in English

8. Published between the years of 1946 and 2019.
Exclusion criteria consisted of studies of patient populations with significant medical comorbidities (i.e. patients with depression post-myocardial infarction), people under age 18 , use of a technology that did not involve video (i.e. telephone-based intervention), and a design that was not an RCT.

\section{Information Sources}

A literature search for terms related to depression and telepsychiatry was conducted by one of the authors $(\mathrm{SH})$ for the following databases: E-publications ahead of print, in-process and other non-indexed citations, Ovid Medline Daily, Ovid Medline, Embase Classic + Embase, CINAHL, PsychINFO, and Scopus.

\section{Search}

We employed the following search strategies: (Telemedicine/ or Telemedicine.mp. or Tele-medicine.mp. or Telepsychiatr*.mp. or Tele-psychiatr*.mp. or Telehealth.mp. or Telehealth.mp. or Tele-health.mp. or Mobile Health.mp. or Mhealth.mp. or M-health.mp. or Ehealth.mp. or E-health. mp. or Remote medicine.mp. or Virtual medicine.mp.) and (exp Depressive Disorder/or Dysthymic Disorder*.mp. or Depressive Neuros?s.mp. or Depressive Syndrome*.mp. or Unipolar Depression*.mp. or Melancholia*.mp. or Endogenous Depression*.mp. or Neurotic Depression*.mp. or Depressive Personality Disorder*.mp. or Clinical Depression*.mp.).

We examined the following outcomes:

1. Acceptability and patient satisfaction

2. Efficacy

3. Cost-effectiveness

\section{Study Selection}

Two study authors (GG and JM) independently went through these citations individually and determined which would be potentially appropriate to include, based on the above criteria. Both authors then met to discuss any discrepancies and determine if a study should be included or not. Disagreement was solved by consensus. The authors' initial intention was to conduct a meta-analysis, although unfortunately the data did not allow for this level of analysis; please see the discussion below for full details regarding why we instead opted for a systematic review. Each included study was read by author JM and checked by author GG, and any information regarding the above three outcomes was included in the systematic review. CB provided overall supervision of the project. 


\section{Results}

The search resulted in 2682 citations, and after removing duplicates, a total of 2170 potentially relevant citations. After reading the abstracts, we excluded 2140 records that were not appropriate because they were: about unrelated topics or had populations with prominent medical comorbidities (913), letters to the editor (40), for the child and adolescent population (112), about other psychiatric conditions but not depression (319), about depression but not involving televideo (620), or about depression and televideo but not an RCT (136). This resulted in 30 studies, for which we then found the full texts. We then narrowed the search to specifically RCTs involving psychiatric care that involved some form of tele-technology, which resulted

Fig. 1 Flow diagram for systematic review in 14 studies. Please see Fig. 1 for the study selection diagram.

Some studies among the ones selected were secondary analyses from the same sample. We grouped the studies based on the same research population (see Table 1), as it was clearly indicated in the papers. We identified 7 samples groups in 14 studies.

See Table 1 for study characteristics, populations, interventions, sample size, length of follow up, measures and study outcomes.

\section{Acceptability and Patient Satisfaction}

All the studies examining acceptability and patient satisfaction showed that there either was no difference between telepsychiatry and in-person care (Egede et al. 2016; Ruskin
Flow Diagram

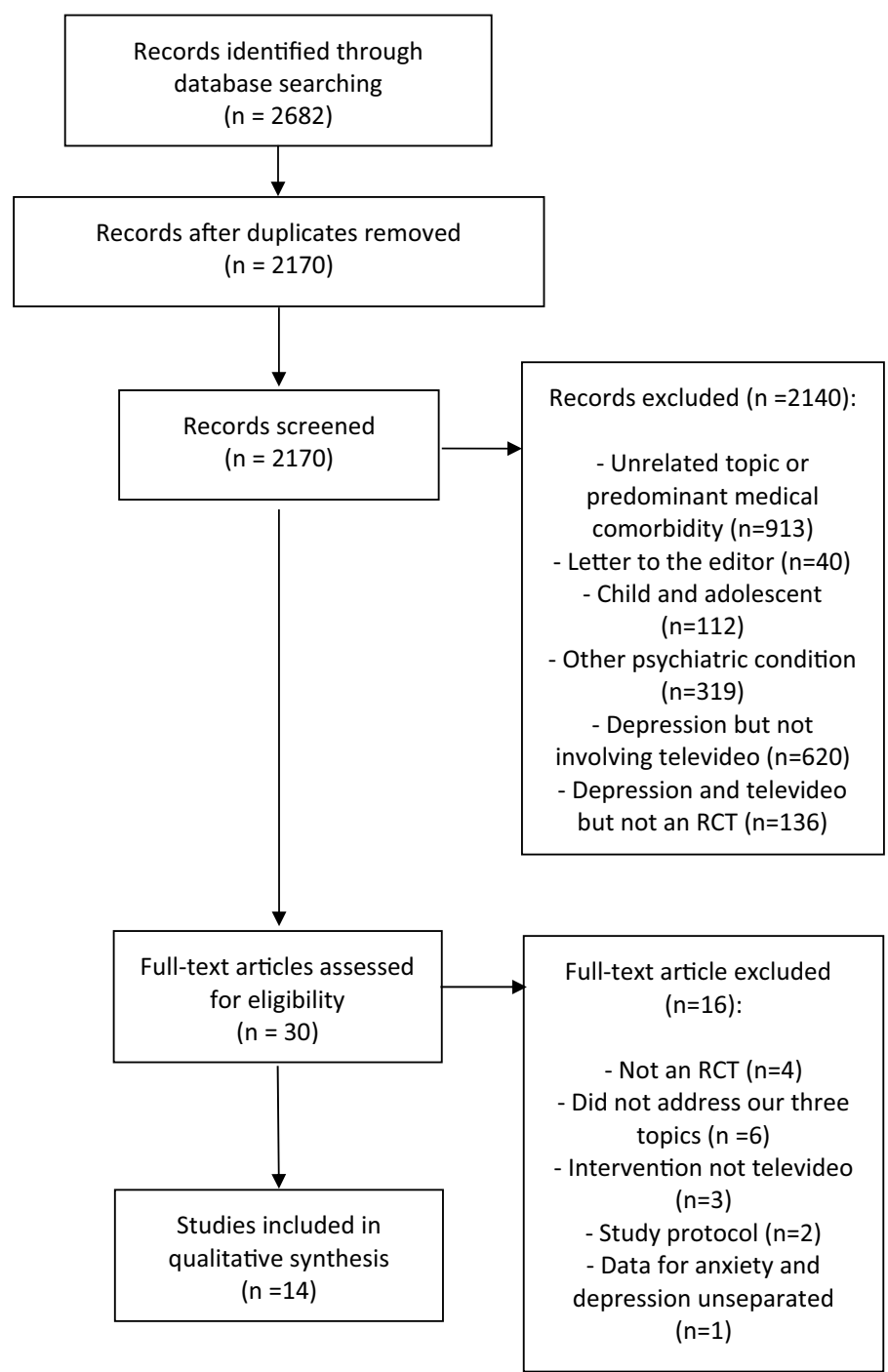




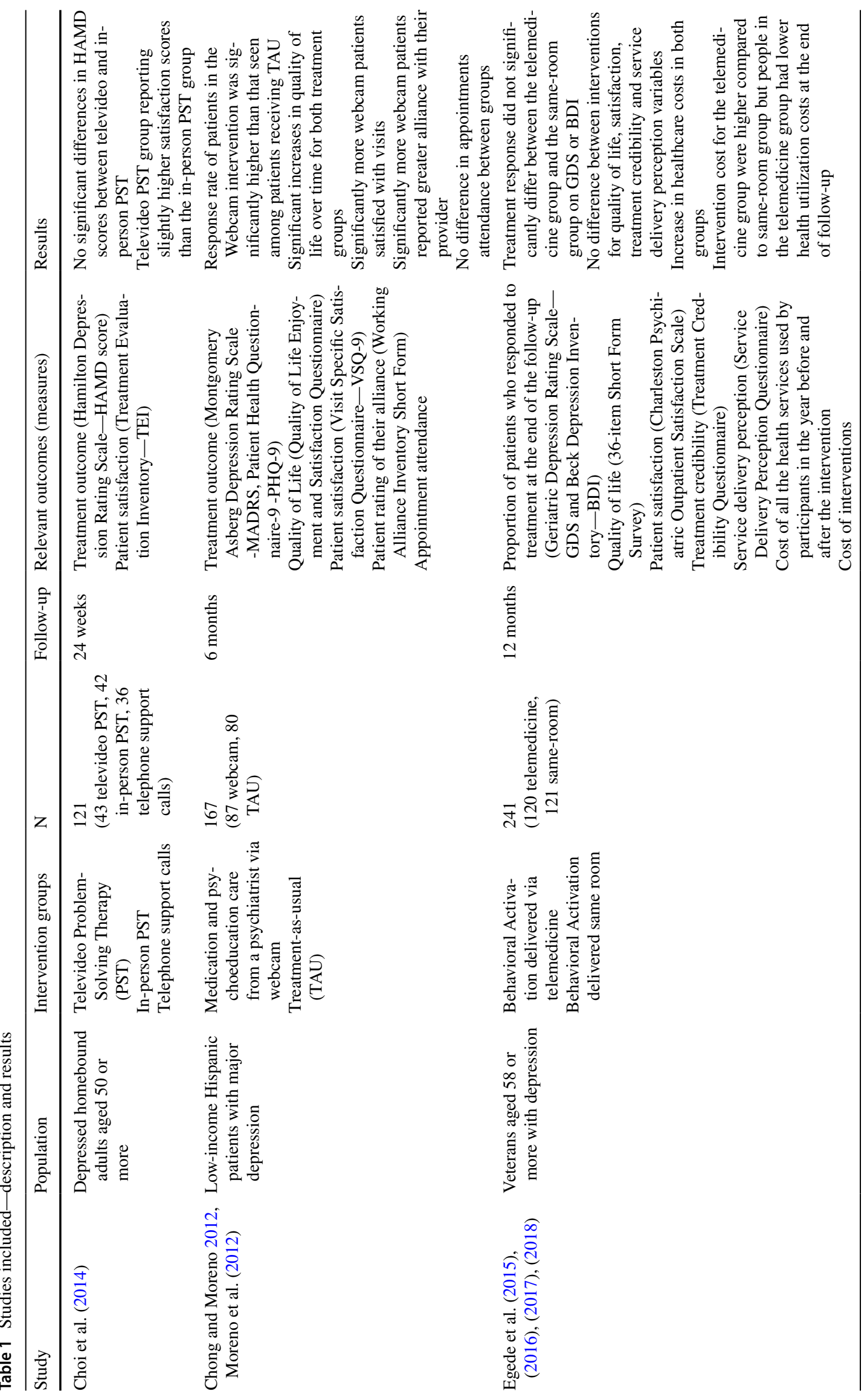




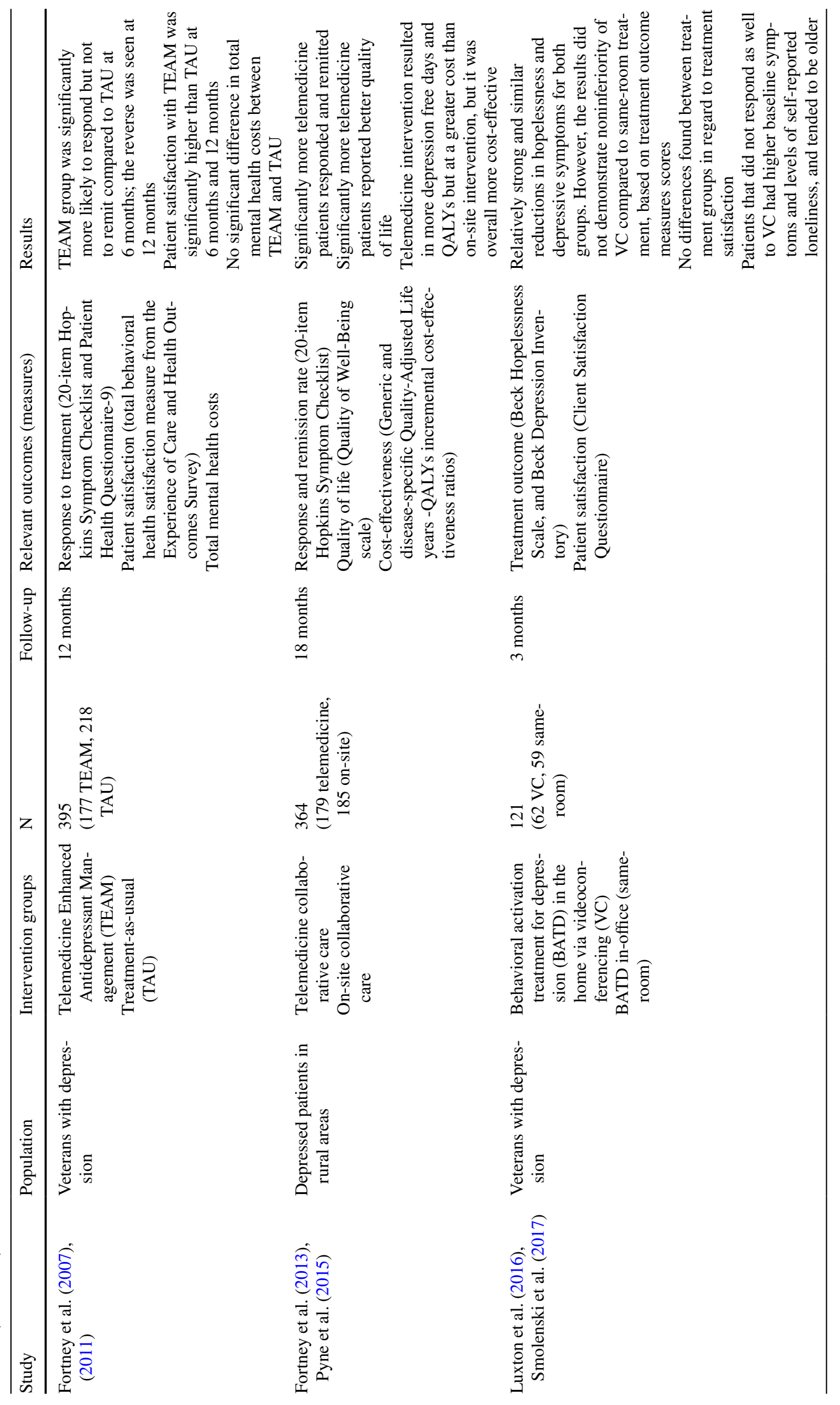




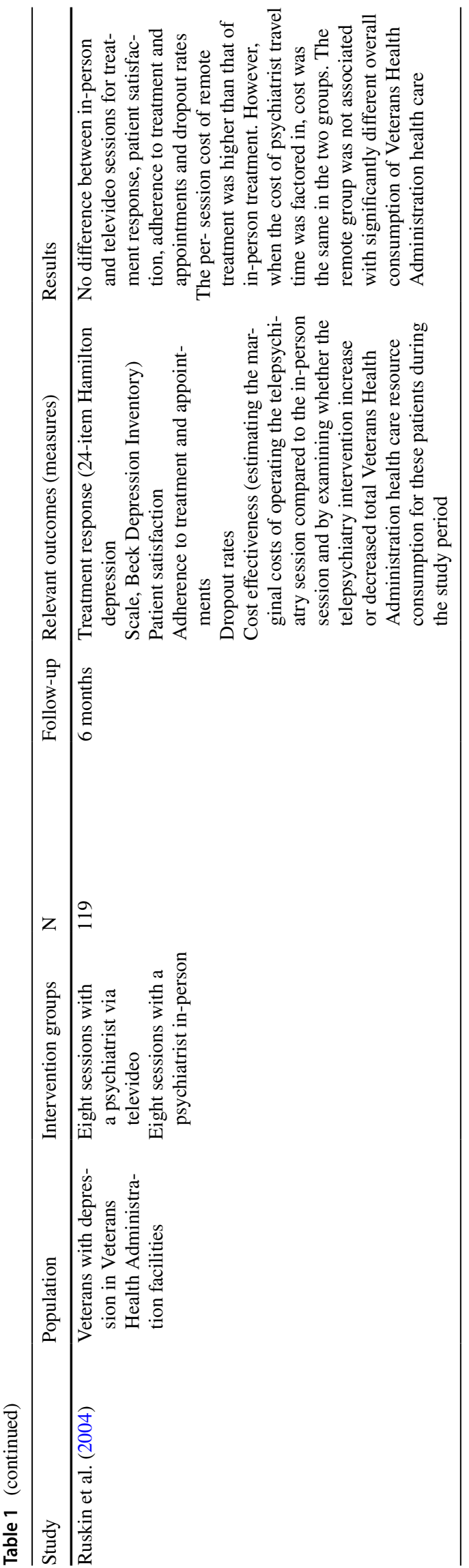

et al. 2004) or patients were more satisfied with telepsychiatry (Chong and Moreno 2012; Fortney et al. 2007; Luxton et al. 2016).

\section{Efficacy}

The vast majority of the studies looking at efficacy, showed that efficacy of treatments has been the same for in-person and telepsychiatry care (Egede et al. 2015; Ruskin et al. 2004) or the telepsychiatry group showed better response to treatment (Choi et al. 2014; Fortney et al. 2007, 2013; Moreno et al. 2012). One study (Luxton et al. 2016) could not reject the null hypothesis that telepsychiatry was no worse than in-person care.

\section{Cost-Effectiveness}

Many of the studies referenced the idea that televideo could be more cost-effective due to reduced travel but did not include it in their analyses (Choi et al. 2014; Chong and Moreno 2012; Moreno et al. 2012). Other studies showed that telepsychiatry was more cost-effective than in-person care (Ruskin et al. 2004; Pyne et al. 2015; Egede et al. 2018) or did not cost more than in-person care (Fortney et al. 2011).

\section{Discussion}

\section{Relevant Findings}

In all of the studies noted, satisfaction (when measured) was either equivalent to face-to-face or significantly higher for all of the groups that included televideo as the intervention. This suggests that, overall, depressed patients find mental health care delivered by televideo to be at least as acceptable as traditional, in-person treatment. This may help ease the concern that patients may find telepsychiatry to be cold or impersonal. In terms of efficacy overall, the studies noted above found that both control and intervention groups for MDD tended to experience relief from depressive symptoms, with the differences between them being either insignificant, or actually in favor of the televideo arm. Despite the increased cost upfront for televideo due to the technology required, all studies cited above found that televideo would eventually be more cost-effective due to time and distance travelled by the patient and/or the practitioner, and thanks to reduced use to healthcare resources overall post-intervention. It is also worth noting that more people now have access to televideo technology via some device of their own, so the costly requirement for a new computer or other device may not be necessary in the future. In addition, the cost of technology overall continues to decrease due to innovation 
in the market, and likely this will result in televideo becoming even more cost-effective in future.

Interestingly, psychiatrists may have a stronger preference for in-person treatment than patients. The RCT by Ruskin et al. (2004) found no difference for patient satisfaction between the two groups, whereas the psychiatrists reported greater satisfaction when seeing patients in person compared to over televideo, although they also tended to fall between agree and strongly agree. Of note, each patient only experienced one interview that was either in-person or over televideo, whereas the psychiatrists all conducted interviews via both methods. However, it suggests that perhaps there is some intangible, difficult to define factor or clinical information that is easier to obtain when face-to-face with the patient; regardless, it does not seem as though its omission negatively impacted patient outcomes (Ruskin et al. 2004).

\section{Link with Other Findings}

The most recent systematic review conducted on key telepsychiatry outcomes across psychiatric disorders, concluded that patients and providers are generally satisfied with telepsychiatry services. Providers, however, tend to express more concerns about the potentially adverse of effects of telepsychiatry on therapeutic rapport. This is in line with our review findings. The authors of the review also state that telepsychiatry is equivalent to in-person assessment in terms of reliability and quality of care, which is, again, similar to what our study has found (Hubley et al. 2016). A previous systematic review on remote treatments for depression was published in 2010 (García-Lizana and Muñoz-Mayorga 2010). The authors mostly include studies where remote technology was used and did not only focus on videoconference. They concluded that videoconference produces the same results as face-to-face treatment and that self-help Internet programs could improve symptoms.

\section{Limitations}

There are a number of limitations regarding our systematic review that make our findings at this time preliminary rather than conclusive. The authors' original intention, as specified in the protocol published on PROSPERO, was to conduct a meta-analysis of the use of telepsychiatry (meaning care provided by a psychiatrist using televideo) in depression for assessment and treatment looking at RCT only. Unfortunately, the only study we found that met all of our inclusion criteria was the RCT by Ruskin et al. (2004), while all of the others could not be included in a meta-analysis. We also attempted to look specifically at patient satisfaction as our primary concern, regardless of which health care provider was involved in the treatment or assessment, so long as it was conducted via televideo. Unfortunately, they all used different means of evaluating patient satisfaction that were difficult to compare, and many of them lacked specific data on standard deviations at baseline and the end of treatment. This may have led to less refined results. Another limitation is that many of these studies were conducted in samples collected entirely from the U.S. Department of Veteran's Affairs (VA); this population is overwhelmingly Caucasian and male, and the results are likely not generalizable to other populations. There is also the strong possibility that publication bias could play a role in our results, as it may be more difficult for a study that was unable to reject its null hypothesis to be published by a reputable journal.

\section{Conclusions}

In terms of future directions for this field, we would urge researchers to consider more closely the impact of the technology acting as the conduit for the therapeutic relationship on the psychiatrist's ability to diagnose and treat MDD. There has been much study of the common factors of psychotherapy as a crucial foundation for a therapeutic relationship, including empathy, alliance, positive regard, and genuineness (Wampold 2015); we do not at this time find any evidence to suggest that these qualities can or cannot be transmitted over video, and we feel this warrants further investigation with more RCTs. We also feel it would be important to conduct more studies similar to Ruskin et al. (2004) on a larger scale that directly compares the same type of psychiatric care delivered in-person or over televideo. Smolenski et al. (2017) observed that patients with milder symptoms and less self-reported loneliness may see more benefit from televideo, so further studies could be done to determine which population benefits most from which modality. It is also important to consider if particular psychiatric conditions or diagnoses may be better or worse served by televideo. Serhal et al. (2017) conducted a cross-sectional study for the fiscal year 2012 to 2013 to determine how many patients and psychiatrists were using televideo in Ontario, and their results were disheartening. Overall, only $7 \%$ of the psychiatrists working in Ontario that year saw patients via televideo (Serhal et al. 2017). There is clearly a great need for many of our most-vulnerable community members to access psychiatry, and yet barriers remain that prevent them from doing so. Perhaps psychiatrists and patients both would feel more comfortable providing and receiving care over televideo if further research could more clearly indicate its acceptability, efficacy, and cost-effectiveness compared to regular, face-to-face care.

It is our hope that further research can lead to more people receiving the care they need and meeting their treatment goals, whether it was conducted over a coffee table or a video screen. 
Funding This research received no specific grant from any funding agency in the public, commercial, or not-for-profit sectors.

\section{Compliance with Ethical Standards}

Conflict of interest The authors declare that there is no conflict of interest.

\section{References}

Canadian Network for Mood and Anxiety Treatments (CANMAT). (2016). Clinical guidelines for the management of adults with major depressive disorder: Disease burden and principles of care. Canadian Journal of Psychiatry, 61(9), 510-523.

Cevik, M., Bamford, C. G. G., \& Ho, A. (2020). COVID-19 pandemic-a focused review for clinicians. Clinical Microbiology \& Infection, 26(7), 842-847.

Chaet, D., Clearfield, R., Sabin, J. E., et al. (2017). Ethical practice in telehealth and telemedicine. Journal of General Internal Medicine, 32(10), 1136-1140.

Choi, N. G., Marti, C. N., Bruce, M. L., et al. (2014). Six-month postintervention depression and disability outcomes of in-home telehealth problem-solving therapy for depressed, low-income homebound older adults. Depress Anxiety, 31, 653-661.

Chong, J., \& Moreno, F. (2012). Feasibility and acceptability of clinic-based telepsychiatry for low-income Hispanic primary care patients. Telemedicine and e-Health, 18(4), 297-304.

Egede, L. E., Acierno, R., Knapp, R. G., et al. (2015). Psychotherapy for depression in older veterans via telemedicine: A randomized, open-label, non-inferiority trial. Lancet Psychiatry, 2, 693-701.

Egede, L. E., Acierno, R., Knapp, R. G., et al. (2016). Psychotherapy for depression in older veterans via telemedicine: Effect on quality of life, satisfaction, treatment credibility, and service delivery perception. Journal of Clinical Psychiatry, 77(12), 1704-1711.

Egede, L. E., Gebregziabhera, M., Walker, R. J., et al. (2017). Trajectory of cost overtime after psychotherapy for depression in older Veterans via telemedicine. Journal of Affective Disorders, 207, $157-162$.

Egede, L. E., Dismuke, C. E., Walker, R. J., et al. (2018). Cost-effectiveness of behavioural activation for depression in older adult veterans: In person-care versus telehealth. The Journal of Clinical Psychiatry, 79(5), e1-e8.

Fortney, J. C., Pyne, J. M., Edlund, M. J., et al. (2007). A randomized trial of telemedicine-based collaborative care for depression. Journal of General Internal Medicine, 22(8), 1086-1093.
Fortney, J. C., Maciejewski, M. L., Tripathi, S. P., et al. (2011). A budget impact analysis of telemedicine-based collaborative care for depression. Medical Care, 49(9), 872-880.

Fortney, J. C., Pyne, J. M., Mouden, S. B., et al. (2013). Practicebased versus telemedicine-based collaborative care for depression in rural federally qualified health centers: A pragmatic randomized comparative effectiveness trial. American Journal of Psychiatry, $170,414-425$.

García-Lizana, F., \& Muñoz-Mayorga, I. (2010). Telemedicine for depression: A systematic review. Perspect in Psychiatr Care, 46(2), 119-126

Hubley, S., Lynch, S. B., Schneck, C., et al. (2016). Review of key telepsychiatry outcomes. World Journal of Psychiatry, 6(2), 269-282.

Lambert, K., \& Wertheimer, M. (2016). Telepsychiatry: Who, what, where, and how. Psychiatric News. https://doi.org/10.1176/appi. pn.2016.2a13.

Luxton, D. D., Pruitt, L. D., Wagner, A., et al. (2016). Home-based telebehavioral health for U.S. military personnel and veterans with depression: A randomized controlled trial. Journal of Consulting and Clinical Psychology, 84(11), 923-934.

Moreno, F. A., Chong, J., Dumbauld, J., et al. (2012). Use of standard webcam and internet equipment for telepsychiatry treatment of depression among underserved Hispanics. Psychiatric Services, 63(12), 1213-1217.

Ontario Telemedicine Network. (2018). OTN Annual report 2017/18. Retrieved April 7, 2019, from https://otn.ca/wp-content/uploa ds/2017/11/otn-annual-report.pdf.

Pyne, J. M., Fortney, J. C., Mouden, S., et al. (2015). Cost-effectiveness of on-site versus off-site collaborative care for depression in rural FQHCs. Psychiatric Services, 66(5), 491-499.

Royal Australian and New Zealand College of Psychiatrists (RANZCP). (2013). Telepsychiatry: RANZCP telehealth survey results. Retrieved October 20, 2019, from https://www.ranzcp.org/files /resources/practice-resources/telehealth-survey-results-v11-(2). aspx.

Ruskin, P. E., Silver-Aylaian, M., Kling, M. A., et al. (2004). Treatment outcomes in depression: Comparison of remote treatment through telepsychiatry to in-person treatment. American Journal of Psychiatry, 161, 1471-1476.

Serhal, E., Crawford, A., Cheng, J., et al. (2017). Implementation and utilisation of telepsychiatry in Ontario: A Population-based study. Canadian Journal of Psychiatry, 62(10), 716-725.

Smolenski, D. J., Pruitt, L. D., Vuletic, S., et al. (2017). Unobserved heterogeneity in response to treatment for depression through videoconference. Psychiatric Rehabilitation Journal, 40(3), 303-308.

Wampold, B. E. (2015). How important are the common factors in psychotherapy? An update. World Psychiatry, 14(3), 270-277.

Publisher's Note Springer Nature remains neutral with regard to jurisdictional claims in published maps and institutional affiliations. 